\title{
Estruturando Desenvolvimento de Software como um Serviço de TI: Uma Experiência Prática
}

\author{
Marcos Kalinowski ${ }^{1}$, Sheila Reinehr ${ }^{2}$ \\ ${ }^{1}$ Programa de Pós-Graduação em Ciência da Computação - Universidade Federal de \\ Juiz de Fora (UFJF) - Rua José Kelmer, s/n - 36.036-330 - Juiz de Fora - MG - Brasil \\ ${ }^{2}$ Programa de Pós-Graduação em Informática - Pontifícia Universidade Católica do \\ Paraná (PUCPR) - Rua Imaculada Conceição, 1155 - 80.215-901 - Curitiba - PR - Brasil \\ kalinowski@ice.ufjf.br, sheila.reinehr@pucpr.br
}

Resumo. Em ambientes organizacionais envolvendo sistemas em produção nem sempre é possivel prever as demandas de desenvolvimento que irão surgir. Neste cenário, torna-se um diferencial para fornecedores estruturar-se para prestar serviços capazes de tratar estas demandas, respeitando, tanto o volume, quanto a prioridade de atendimento. Neste artigo é relatada a experiência de estruturar o desenvolvimento de software na forma de prestação de um serviço de TI que atenda a um acordo de nível de serviço (SLA) estabelecido com o cliente para atender às suas necessidades. Serão apresentadas a visão externa, por meio dos aspectos contratuais, e a visão interna, por meio dos controles gerenciais utilizados para assegurar o atendimento do SLA.

\begin{abstract}
In organizational environments involving systems in production, it is not always possible to predict development demands that will arise. In this scenario, it becomes a business advantage for suppliers to structure themselves in order to provide services able to deal with these demands, properly handling their volume and their treatment priority. In this paper, we report the experience of structuring software development as an IT service delivery according to a service level agreement (SLA) established along with the customer to meet their business needs. Both, the external vision, through contractual terms, and the internal vision, through managerial controls used to meet the SLA, are presented.
\end{abstract}

\section{Introdução}

Em ambientes organizacionais dinâmicos, envolvendo sistemas em produção, nem sempre é possível prever e formalizar contratualmente as demandas de desenvolvimento que irão surgir. Desta forma, é comum que organizações busquem parceiros de Tecnologia da Informação (TI) que possam prestar serviços de desenvolvimento para tratar suas demandas na medida em que elas surgem, em função de suas reais necessidades, respeitando, tanto a variação no volume destas demandas, quanto a prioridade para seu atendimento. Entretanto, sob o ponto de vista do fornecedor, estruturar o desenvolvimento de software de modo a atender a estas expectativas não é uma tarefa trivial.

Acredita-se que uma das formas de atender a estas expectativas seja estruturar o atendimento das demandas de desenvolvimento por meio do estabelecimento de 
capacidades de gerenciamento de serviços, onde cada demanda passaria a ser tratada como uma solicitação de um serviço de TI. Tratar o atendimento às demandas de desenvolvimento como um serviço prestado ao cliente está aderente à definição de serviços da (ISO/IEC, 2011), que define um serviço como "um meio de entregar valor para o cliente por meio da facilitação de resultados que o cliente deseja atingir".

O gerenciamento de serviços de TI, por sua vez, pode ser definido de acordo com o ITIL (Information Technology Infrastructure Library), um modelo concebido pelo governo britânico visando trazer um consenso sobre as melhores práticas para o gerenciamento de serviços de TI, como "Um conjunto de capacidades organizacionais especializadas para prover valor aos clientes na forma de serviços" (TSO, 2011).

Este artigo relata a experiência de estruturar o desenvolvimento de software na forma de prestação de serviços de TI, seguindo preceitos do modelo MPS-SV (SOFTEX, 2012) para estabelecer capacidades de gerenciamento das solicitações de serviços (demandas de desenvolvimento) visando atender a um acordo de nível de serviço (Service Level Agreement - SLA) definido com o cliente para atender às suas necessidades.

Serão relatados tanto aspectos contratuais desta estruturação, quanto controles internos empregados visando assegurar o atendimento do SLA, incluindo o uso integrado de planilhas gerenciais, sistemas de issue tracking e quadros kanban para controlar a priorização e o atendimento das demandas.

O restante deste artigo está organizado da seguinte forma. Na seção 2, o contexto da experiência é descrito. Na seção 3, a experiência de estruturar o desenvolvimento de software como um serviço de TI é relatada. Na seção 4 uma análise da experiência é apresentada, destacando dados quantitativos, qualitativos e lições aprendidas. Por fim, na seção 5, as considerações finais são descritas.

\section{Contexto}

A experiência ocorreu no contexto de desenvolvimento de software por parte da Kali Software para a Tranship Transportes Marítimos. A Kali Software atuou com desenvolvimento sob encomenda durante quase uma década (Novembro de 2004 à Março de 2013), prestando serviços para clientes de diferentes áreas de negócio, tanto do Brasil, quanto do exterior. A Tranship é uma empresa brasileira de navegação que presta serviços estratégicos ao país, incluindo serviços de apoio marítimo a plataformas de exploração de petróleo no contexto do pré-sal, entre outros.

A parceria para desenvolvimento de software entre as empresas se estabeleceu em Fevereiro de 2011 e se encerrou em função da decisão da Kali Software de suspender suas atividades em Março de 2013. O software desenvolvido foi um sistema de gestão integrada (Enterprise Resource Planning - ERP), envolvendo diversos módulos (incluindo o módulo administrativo, de operações, de alocação de tripulantes, financeiro, recursos humanos, entre outros) que foram gradualmente postos em produção. Ao todo, 87 casos de uso foram implementados, resultando em um sistema de gestão com 82.931 linhas de código Java, manipulando 167 tabelas.

Com a entrada dos módulos em produção, as necessidades do cliente foram mudando, as diretorias (executiva, de operações, financeira e de recursos humanos) passavam a precisar de informações e novas funcionalidades em curtos intervalos de tempo, de acordo com as necessidades estratégicas, táticas e operacionais de cada área. 
O modelo contratual de desenvolvimento sob encomenda de demandas previamente agrupadas e dimensionadas parecia não se adequar mais ao novo cenário.

Assim, as diretorias das empresas se reuniram para definir um novo modelo contratual de atendimento às demandas. Optou-se pelo formato de prestação de serviços, em que as demanda fossem tratadas como solicitações de serviços e o seu atendimento e faturamento estivessem vinculados ao cumprimento de um SLA. O novo modelo implicou em se estabelecer uma estratégia de entrega de serviços que envolvesse controles internos para facilitar a gerência das solicitações de modo a atender ao SLA.

\section{Estruturando Desenvolvimento de Software como um Serviço}

Embora uma avaliação oficial não estivesse nos planos iniciais desta experiência, a estruturação do desenvolvimento de software como um serviço seguiu preceitos do nível G do modelo de referência MPS-SV (SOFTEX, 2012), de modo que boas práticas previstas por este modelo fossem incorporadas na estruturação. Acredita-se que, assim como o MPS-SW tem promovido a adoção de boas práticas pela indústria de software brasileira (Kalinowski et al., 2010), também o MPS-SV possibilite promover a adoção de boas práticas pela indústria para o fornecimento de serviços de TI.

O nível G do MPS-SV envolve cinco processos: Gerência de Trabalhos, Gerência de Requisitos, Entrega de Serviço, Gerência de Nível de Serviço e Gerência de Incidentes. Assim, no contexto desta experiência, buscou-se complementar práticas de Gerência de Trabalhos e de Requisitos, já estabelecidas na Kali Software no contexto de desenvolvimento de software, com práticas previstas nos demais processos.

O processo de Entrega de Serviços visa a estabelecer uma estratégia de entrega de serviços em conformidade com os acordos de serviço. A Gerência de Nível de Serviços, por sua vez, visa a garantir que os acordos de nível de serviço para cada cliente sejam atendidos. Por fim, o propósito do processo de Gerência de Incidentes, envolve o gerenciamento de incidentes e solicitações de serviços (SOFTEX, 2012).

A Entrega de Serviços é tratada na próxima subseção (3.1), em que os aspectos contratuais que viabilizaram pôr em prática a estratégia de entrega do serviço e o SLA definido para atender às necessidades do cliente serão descritos. A Gerência de Nível de Serviços e a Gerência de Incidentes são tratadas na subseção 3.2, em que os controles gerenciais internos empregados para permitir monitorar o cumprimento do SLA e operacionalizar o atendimento às solicitações de serviço são relatados.

\subsection{Aspectos Contratuais}

O modelo contratual precisou passar por mudanças para viabilizar a nova estratégia de entrega de serviços, em que demandas de desenvolvimento passariam a ser tratadas como solicitações de serviços. Antes, as demandas eram agrupadas em incrementos tratados como projetos de desenvolvimento, faturados de acordo com o seu progresso ( $20 \%$ no início, $30 \%$ após a especificação funcional e $50 \%$ após a entrega final).

Com a nova dinâmica pretendida, as solicitações não seriam mais agrupadas em projetos de desenvolvimento, mas, sim, tratadas como trabalhos associados a serviços isolados que deveriam atender a um SLA. O CMMI-SVC (SEI, 2010) define um trabalho como "um conjunto gerenciado de pessoas e outros recursos alocados que entregam um ou mais serviços para um cliente ou usuário final". A migração do gerenciamento de projetos para o gerenciamento de trabalhos implicou em uma granularidade menor dos itens a serem gerenciados. 
A estratégia definida para viabilizar a entrega dos serviços envolveu dividir o modelo de faturamento do contrato em duas partes, uma fixa e uma variável. A parte fixa envolvia internalizar um analista de requisitos no cliente, responsável por receber demandas dos usuários, e especificá-las como solicitações de serviços. Além do analista internalizado, a parte fixa compreendia também um valor para serviços de manutenção corretiva. A parte variável mensal, por outro lado, era calculada em função do atendimento das solicitações de acordo com o SLA definido com o cliente. A estratégia de atendimento às demandas do cliente está ilustrada na Figura 1.

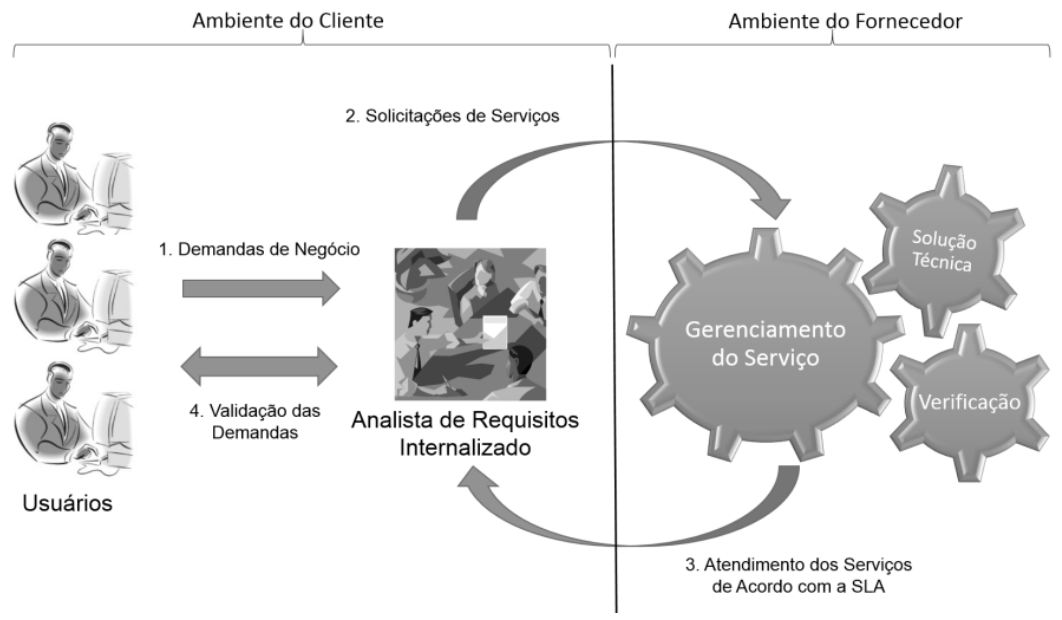

Figura 1. Estratégia de Atendimento às Demandas do Cliente.

O acordo de nível de serviço, cujo atendimento deveria ser gerenciado, definido também como parte do contrato encontra-se na Figura 2.

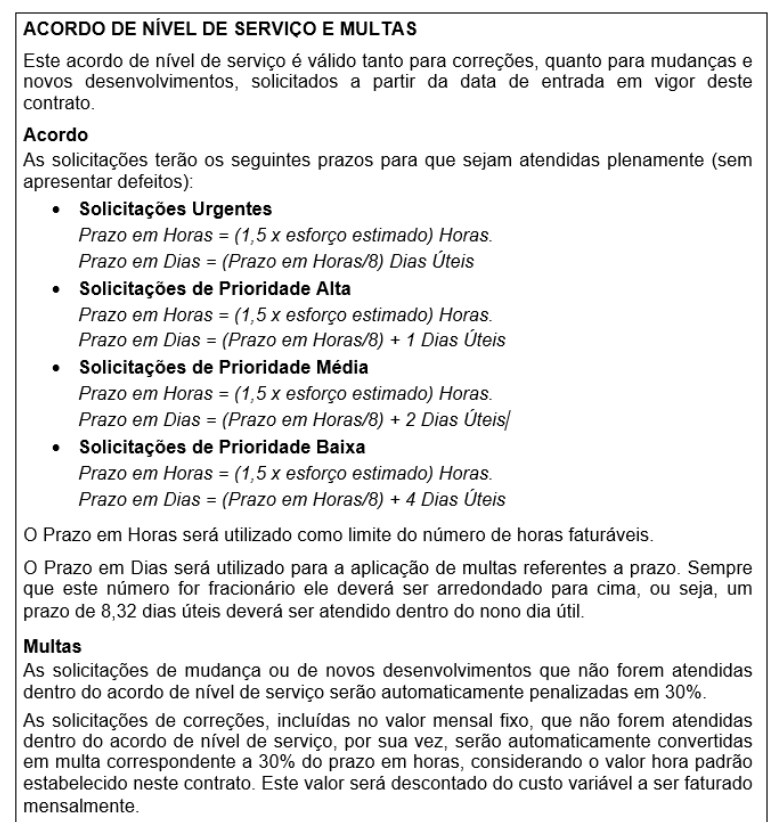

Figura 2. Acordo de Nível de Serviço.

Como pode ser visto, solicitações possuem diferentes prazos, definidos em dias para seu atendimento, em função de sua prioridade. O seguinte critério de priorização 
foi adotado:

- Solicitações Urgentes: impedem o uso do sistema para a realização das atividades de algum dos setores.

- Solicitações de Prioridade Alta: não afetam o funcionamento do sistema, mas impedem a conclusão de alguma demanda vinculada ao sistema.

- Solicitações de Prioridade Média: não afetam o funcionamento do sistema, mas permitem a execução mais célere de alguma demanda vinculada ao sistema.

- Solicitações de Prioridade Baixa: não afetam o funcionamento do sistema, mas permitem aperfeiçoar a sua funcionalidade.

Para permitir monitorar o atendimento do SLA, na medida em que surgiam, as solicitações eram registrados em uma planilha de controle de SLA, um dos controles gerenciais internos empregados para facilitar o gerenciamento dos serviços. Mais sobre os controles gerenciais internos será relatado na próxima subseção.

\subsection{Controles Gerenciais Internos}

Os controles empregados para apoiar o gerenciamento dos serviços envolveram uma planilha de controle de SLA, um sistema de issue tracking (integrado com controle de versões) e um quadro Kanban para explicitar o status do atendimento a cada instante para toda a equipe envolvida na prestação do serviço. Quadros Kanban tem sido utilizados em abordagens ágeis de desenvolvimento como Lean e SCRUM, entre outros.

A sequência em que estes controles são acionados para atender a uma nova solicitação de serviços está ilustrada na Figura 3. A figura destaca também o propósito e as facilidades oferecidas por cada um destes controles.

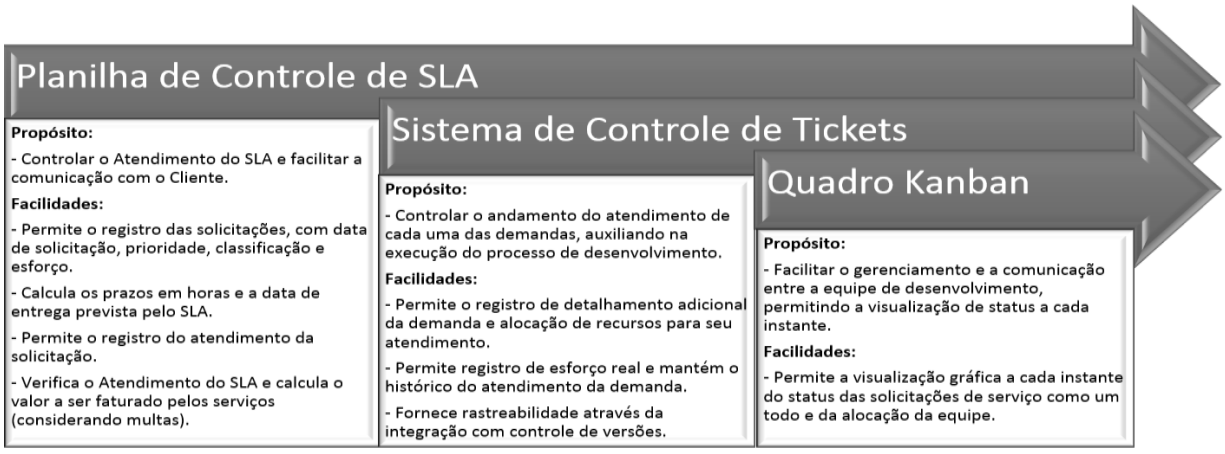

Figura 3. Controles Internos para o Gerenciamento do Serviço.

Inicialmente a solicitação é registrada na planilha. Um recorte desta planilha, contemplando solicitações feitas entre 16/01/2013 e 31/01/2013 encontra-se na Figura 4. As colunas referentes ao desenvolvedor e ao custo foram propositalmente modificadas para não expor o desempenho individual nem os preços praticados. Assim, o custo foi modificado para conter número de horas faturáveis ao invés de valores.

Como pode ser visto, os dados registrados para cada solicitação são: prioridade, data da solicitação, tipo (correção ou mudança), data da abertura do ticket, número do ticket (para acompanhamento no sistema de issue tracking), módulo, caso de uso e esforço estimado. A partir destas informações, o prazo em horas e a data de entrega prevista são calculados de acordo com o SLA. A primeira coluna destaca a situação gerencial, mostrando se a solicitação foi entregue, cancelada ou quantos dias faltam para a entrega prevista. 


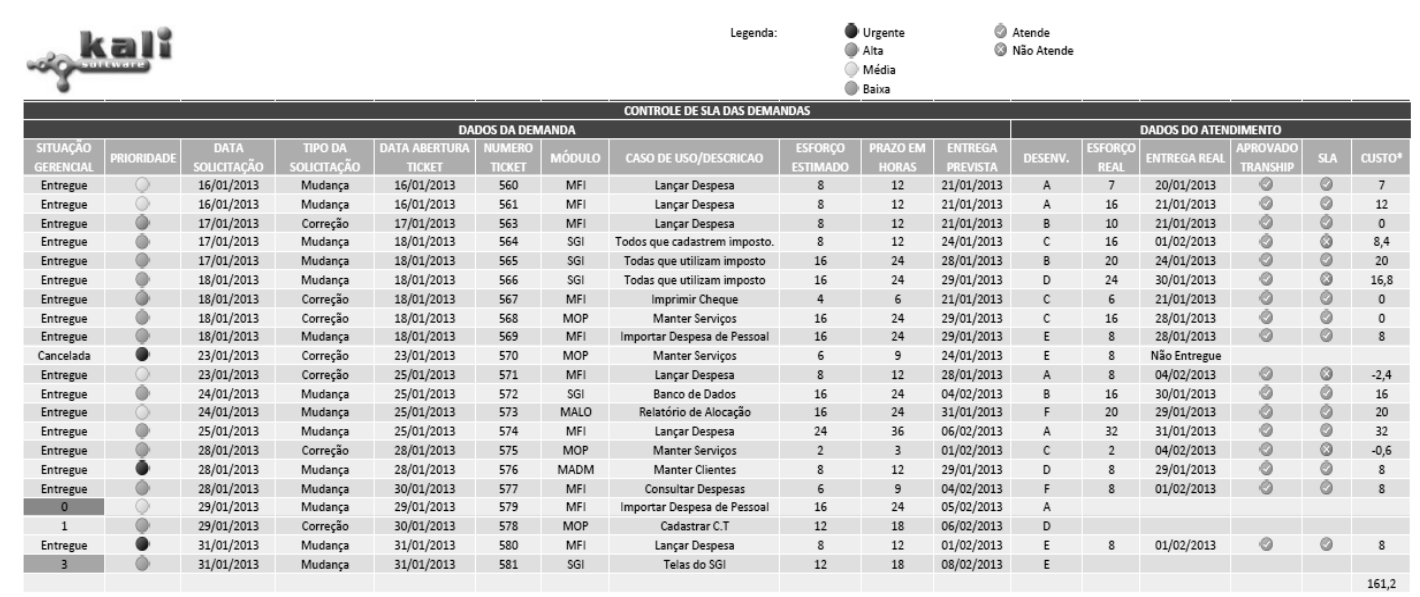

Figura 4. Planilha de Controle de SLA

Os dados do atendimento da solicitação, também contidos na planilha, envolvem o desenvolvedor, o esforço real (obtido do sistema de issue tracking), a data da entrega real e a aprovação pelo cliente (validação). Com estas informações a planilha calcula se o SLA foi atendido e o valor a ser faturado pelo atendimento, já considerando multas por atraso, se for o caso. Nestes 15 dias, a planilha informa que há 161,2 horas faturáveis (referentes a mudanças, correções estão no custo fixo).

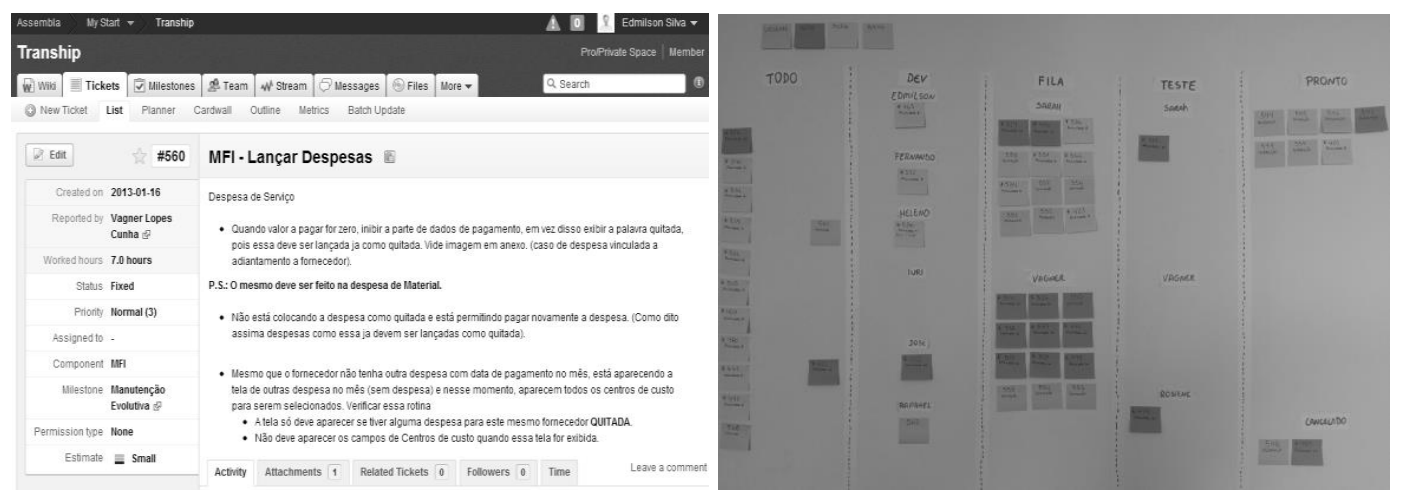

Figura 5. Sistema de issue tracking e quadro Kanban

O sistema de issue tracking utilizado foi o Assembla, que já contempla a integração de tickets (tarefas) com o controle de versões. Nele são alocados os desenvolvedores e é realizado o registro do esforço real. Assim que um desenvolvedor conclui a solução técnica de uma solicitação, antes que ela seja dada como atendida, o ticket é passado para testes por uma equipe independente. $\mathrm{O}$ controle do status de atendimento de cada solicitação fica facilitado por um quadro Kanban exposto fisicamente na sala da equipe. Um exemplo real de uma solicitação no Assembla e uma foto do quadro Kanban expondo o status das solicitações podem ser vistos na Figura 5.

A importância dos testes nesta estruturação é evidente, uma vez que uma solicitação só é aceita para faturamento se estiver sem defeitos. Inspeções de requisitos (Kalinowski et al., 2007) e revisões por pares no código foram também adotadas.

\section{Análise da Experiência}

Os elementos principais deste relato de experiência foram a estruturação do 
desenvolvimento de software como um serviço de TI e controles adotados para o gerenciamento deste serviço, utilizando conceitos de agilidade. Assim, a análise se baseará em dados quantitativos e qualitativos, referentes a resultados obtidos em relação aos pilares do gerenciamento (qualidade, prazo e custo) e percepções e lições aprendidas desta nova estruturação.

Dados quantitativos foram obtidos da comparação com a entrega anterior à reestruturação, em que se adotava desenvolvimento sob encomenda no formato de projeto. Foram considerados apenas desenvolvimentos de casos de uso novos de complexidade média (seguindo o critério da contagem de pontos de caso de uso - de 4 a 7 transações incluindo passos alternativos). O comparativo está ilustrados na Tabela 1.

Tabela 1. Comparativo da Reestruturação com o Formato Anterior

\begin{tabular}{|l|c|c|c|}
\hline Abordagem & \# Casos de Uso & \# Defeitos (Aceitação) & Esforço \\
\hline Projeto & 12 & 4 & 284 \\
\hline Serviço de TI & 11 & 2 & 232 \\
\hline
\end{tabular}

Em relação à qualidade, foi possível notar uma redução no número de defeitos nos testes de aceitação do cliente por caso de uso de complexidade média, de 0,33 defeitos por caso de uso para 0,18 . O esforço real gasto na implementação (que pode ser indiretamente associado tanto a prazo, quanto a custo) também foi menor. Houve uma redução do esforço de 23,66 horas por caso de uso para 21,09.

É evidente que estas diferenças não são estatisticamente significativas e que há diversos fatores que podem ter introduzido viés nestes resultados. Entretanto, foi recebido retorno também do cliente relatando menos problemas nos testes de aceitação e maior agilidade no atendimento às demandas. A percepção geral foi a de ter estabelecido um modelo mais flexível para atender às necessidades do cliente (não precisa ser refeito em função de uma variação no volume de solicitações, por exemplo) dentro de suas expectativas de fornecimento (expressas através do SLA).

Uma sessão informal de análise causal (Kalinowski et al., 2012) referente a estas mudanças apontou que a existência do SLA e a gerência mais próxima resultaram em um aumento do comprometimento dos desenvolvedores. Alguns mencionaram que, sabendo da existência de multas individuais por solicitação para a empresa, eles viam não atender ao SLA de forma muito mais severa do que ultrapassar prazos previstos no cronograma do modelo anterior. De fato, gerenciar de forma mais próxima e com uma granularidade menor são sugestões contidas nas reflexões de Humphrey a respeito de gerenciamento eficiente de equipes (Humphrey, 2010).

Entre as lições aprendidas que podem ser passadas para empresas que busquem fornecer desenvolvimento no formato de um serviço de TI, pode-se destacar:

- Neste modelo de fornecimento é importante que se tenha uma folga orçamentária na parte mensal fixa do contrato, compensando possíveis variações de volume de solicitações a serem faturadas na parte mensal variável.

- É importante que se avalie o SLA cuidadosamente, verificando se há capacidade instalada para realmente atender às solicitações neste nível de serviço.

- O critério de priorização das solicitações deve ser explícito, pois sua aplicação pode-se mostrar útil para evitar multas indevidas associadas ao SLA.

- Alguns colaboradores se sentiram pressionados pela existência do SLA e o monitoramento mais próximo e quantitativo. Assim, pode ser interessante estabelecer uma política complementar de bônus por valor agregado ao cliente.

- O esforço da reestruturação foi baixo, tendo ocorrido dentro de um mês. Foram 
percebidos benefícios em relação à qualidade, prazo, custo e satisfação do cliente. Como a reestruturação tratou aspectos de todos os processos do nível $\mathrm{G}$ do modelo MPS-SV, acredita-se que implantar este modelo possa envolver pouco esforço e resultar em um aumento considerável da capacidade produtiva.

\section{Considerações Finais}

Neste artigo foi relatada a experiência de estruturar o desenvolvimento de software na forma de prestação de serviços de TI, envolvendo estabelecer capacidades de gerenciamento das solicitações de serviços visando atender a um acordo de nível de serviço estabelecido juntamente com o cliente para atender às suas necessidades.

A estruturação seguiu preceitos do modelo MPS-SV, complementando práticas de engenharia de software já estabelecidas com boas práticas da prestação de serviços de TI e práticas ágeis. Foram relatados, tanto aspectos contratuais da estruturação, quanto controles internos empregados visando assegurar o atendimento do SLA, envolvendo o uso integrado de planilhas gerenciais, sistemas de issue tracking e quadros kanban.

Uma análise da experiência foi descrita, explicitando uma percepção de pouco esforço envolvido na estruturação e de benefícios obtidos em relação à qualidade, prazo, custo e satisfação do cliente. Acreditamos que o relato deste artigo e suas lições aprendidas possam servir como ponto de partida para empresas que queiram se estruturar para fornecer desenvolvimento de software no formato de um serviço de TI, podendo assim se beneficiar da adoção do modelo MPS-SV de forma sinérgica com as práticas de engenharia de software já estabelecidas.

\section{Referências}

Humphrey, W.S. (2010). "Reflections on Management”, Addison-Wesley, 2010 (ISBN 978-85-99334-11-9).

ISO/IEC (2011). "ISO/IEC 20000-1:2011(E) - Information Technology Service Management". Geneve, 2011.

Kalinowski, M., Card, D.N., Travassos, G.H. (2012). "Evidence-Based Guidelines to Defect Causal Analysis", IEEE Software, vol. 29, no. 4, pp. 16-18, July-Aug. 2012, doi: 10.1109/MS.2012.72

Kalinowski, M., Santos, G., Reinehr, S., Montoni, M., Rocha, A. R., Weber, K.C., Travassos, G. H. (2010). "MPS.BR: Promovendo a Adoção de Boas Práticas de Engenharia de Software pela Indústria Brasileira". XIII Congreso Iberoamericano en "Software Engineering" (CIBSE), Cuenca, Equador, 2010.

Kalinowski, M., Spínola, R.O., Dias Neto, A.C., Bott, A., Travassos, G. H. (2007). "Inspeções de Requisitos de Software em Desenvolvimento Incremental: Uma Experiência Prática", VI Simpósio Brasileiro de Qualidade Software (SBQS), Porto de Galinhas - PE, Brasil, 2007.

SEI - Software Engineering Institute (2010). "CMMI-SVC - Capability Maturity Model Integration for Services". Disponível em www.sei.cmu.edu/cmmi

SOFTEX (2012). "MR-MPS-SV - Guia Geral MPS de Serviços". Disponível em www.softex.br/mpsbr

TSO - The Stationery Office (2011). "ITIL - Information Technology Infrastructure Library v2011”. 